Lengua y Sociedad en el Mundo Hispánico

Language and Society in the Hispanic World

Editado por / Edited by

Julio Calvo Pérez (Universitat de València)

Luis Fernando Lara (E1 Colegio de México) Matthias Perl (Universität Mainz)

Armin Schwegler (University of California, Irvine)

Klaus Zimmermann (Universität Bremen)
Yolanda Congosto Martín

Elena Méndez García de Paredes (eds.)

\section{Variación linguiística y contacto de lenguas en el mundo hispánico}

In memoriam MANUEl Alvar

Vol. 27 


\title{
LA PERSPECTIVA VARIACIONISTA EN EL ESTUDIO DE LA (DES)CORTESÍA VERBAL
}

\author{
ESPERANZA R. ALCAIDE LARA \\ Universidad de Sevilla
}

\section{Introducción}

Es una realidad empíricamente observable que en la realización de una lengua se pueden distinguir saberes idiomáticos distintos que se ponen en práctica condicionados por diferentes variables contextuales: el estrato sociocultural al que pertenece el locutor, el sexo, la edad, el tenor interpersonal que se establece entre los interlocutores, el género y modo discursivos, el campo, e incluso la procedencia geográfica del hablante. Esta heterogeneidad, que se da en todos los niveles del lenguaje, afecta igualmente a la expresión de la (des)cortesía, por lo que se hace necesario abordar el estudio de esta función discursiva desde una perspectiva netamente variacionista.

Partimos de la base de que un mismo recurso lingüístico sirve bien para llevar a cabo estrategias de cortesía, bien estrategias de descortesía, dependiendo de todos los factores mencionados anteriormente'.

Por ello, pensamos que el objetivo de los futuros estudios sobre (des)cortesía debe ser lograr establecer el paradigma de recursos disponibles para llevar a cabo

1. Por ejemplo, las llamadas palabras malsonantes pueden ser formas de imposición (descortesía) o de afiliación (cortesía). Su uso y valor son muy variados. Así, generalmente son de escasa utilización en los debates televisivos, salvo en situaciones de crispación y conflicto de grado muy elevado, cuando el tema que centra el debate no es excesivamente serio (por ejemplo, los del "corazón"), los interlocutores no son profesionales de los medios (típicos colaboradores "advenedizos"), las horas de emisión son de las denominadas de "adultos" (noche, preferentemente), y el tenor interpersonal entre los intervinientes es de cercanía y familiaridad. De lo contrario, si una de estas variables decrece se utilizarán otros recursos lingüísticos para llevar a cabo la estrategia descortés de la imposición. 
las distintas estrategias de cortesía o descortesía, dependiendo de todas estas variables ${ }^{2}$.

La mayoría de los actuales trabajos sobre (des)cortesía señalan su carácter cultural. Se habla de una perspectiva no etnocentrista en el estudio de este fenómeno, basada en cómo las diferencias culturales dan lugar a distintas formas de evaluar estrategias como corteses, descorteses, o anticorteses incluso. Y se insiste en la comparación entre distintas culturas en relación con la valoración de las interrupciones, la usurpación de turnos, etc., contraponiendo, sobre todo, culturas nórdicas a culturas mediterráneas. Todo ello indica que la (des)cortesía es, cuando menos, una "categoría vacía" (Bravo 2005), que será rellenada según los parámetros culturales de las distintas comunidades lingüísticas y de habla (Romaine 1996).

En definitiva, la hipótesis que pretendemos defender aquí es que, al igual que cualquier otro aspecto del uso de una lengua es susceptible de ser estudiado desde el punto de vista de la variación lingüística, la (des)cortesía no solo puede, sino que debe ser analizada desde esta perspectiva, en cuanto que es un fenómeno discursivo.

\section{Un poco de historia}

La cortesía empieza a tener un papel relevante en el panorama lingüístico en las primeras propuestas que se orientaban a la preponderancia de lo normativo y lo racional. En esta línea se sitúan Grice $(1967)^{3}$, Lakoff $(1973)^{4}$, Leech $(1983)^{5}$ o Fraser y Nolen $(1981)^{6}$. Las posturas de todos estos autores se enclavan en el período prepragmático del estudio de la cortesía.

A partir de 1987, con las figuras de Brown y Levinson, se abre una nueva era en estos estudios, que Watts (2003) denomina los pospragmáticos. Constituyen el pun-

2. Tal vez una tarea ingente, pero, por ejemplo, de gran rentabilidad para la enseñanza de las lenguas como L2.

3. Máxima de cortesía: be polite.

4. Rules of Pragmatic Competence: sea claro; sea cortés: no se imponga; dé opciones; haga que el destinatario se sienta bien; sea amable.

5. Principio de cortesía (regulador del equilibrio social): máxima de tacto, de generosidad, de aprobación, de modestia, de acuerdo, de consideración o simpatía.

6. Teoría del contrato social: derechos y obligaciones que se van renegociando en el transcurso de la interacción. 
to de inflexión en el análisis de la cortesía, más allá de lo meramente establecido como "buenas maneras". Pese al reconocimiento que prácticamente la totalidad de los analistas les profesan, estos autores han sido objeto de no pocas críticas.

En este momento, lo que más nos interesa es que parten del supuesto de racionalidad en la persona, por el que todos ponemos en funcionamiento una serie de recursos para conseguir unos fines, base de la interacción social. La elección de estos depende de la existencia de unas variables sociológicas como la distancia social entre hablante y oyente, el poder relativo existente entre ellos y el grado de imposición que suponga determinado acto. De la relación de todo ello, resulta el grado de amenaza que supone cada uno de los actos para la imagen del hablante, del oyente o de los dos a la vez. Este es el primer modelo en el que se explicita el condicionante de variables. El inconveniente es que es una tesis etnocentrista; es decir, pretende que los resultados de esa ecuación sean aplicables a todas las situaciones en todas las lenguas y culturas. Pero una teoria que ha sido centrada en el modelo anglosajón dificilmente será de aplicación absoluta en otros entornos como el mediterráneo, por ejemplo, puesto que existen "comunidades de habla", que, sin compartir lenguas, son capaces de compartir comportamientos lingüísticos. E incluso, dentro de una misma lengua, como es el caso del español, podemos distinguir formas de expresar la cortesía y la descortesía distintas. A eso obedecen afirmaciones como "los españoles peninsulares son más directos a la hora de hacer peticiones que los mexicanos", por ejemplo. Es decir, la visión anglosajona, tan pesimista acerca de las relaciones sociales a través del lenguaje, pues parecen estar continuamente en peligro, no vale para otros ámbitos como el español. De hecho, Meier (1995) demuestra cómo la petición, un acto que Brown y Levinson catalogan como altamente amenazante, es un acto de solidaridad en otras culturas $^{7}$. La gran mayoría de los autores actuales han hecho hincapié en la necesidad de una relativización cultural de los conceptos propuestos por estos, que pasa, a juicio de Blum-Kulka (1990), incluso por los propios componentes de las necesidades de imagen de una cultura a otra.

\section{Concepto de (des)cortesía.}

La (des)cortesía es en sí misma una función comunicativa universal, aunque, tal y como han demostrado los más recientes estudios ${ }^{8}$, se manifiesta de modo muy

7. También lo han demostrado para el español Hernández Flores (2002) y Bernal (2007) entre otros.

8. Cfr. Kerbrat-Orecchioni $(1996,2004)$ y Bravo $(1999,2003,2004)$ entre otros. 
distinto en cada cultura, en cada lengua. Nosotros vamos a partir de la definición más comúnmente aceptada en la actualidad:

una actividad comunicativa cuya finalidad propia es quedar bien con el otro y que responde a normas y a códigos sociales que se suponen en conocimiento de los hablantes: Este tipo de actividad en todos los contextos considera el beneficio del interlocutor. E1 efecto que esta actividad tiene en la interacción es interpersonalmente positivo (Bravo 2005: 33-34).

Aunque en nuestra opinión, esta definición adolece de limitaciones. La más relevante en este momento es que solo cubre el campo de la cortesía, y deja de lado lo que es la descortesía propiamente dicha, aspectos que concebimos interrelacionados. Por ello, consideramos la (des)cortesía verbal como una categoría discursiva, por la que el hablante se marca el objetivo de dar una imagen de sí mismo de acuerdo con unos parámetros sociales establecidos y compartidos por los participantes en una interacción verbal. Según esto, la estrategia (des)cortés tendría como finalidad o 1) quedar bien con el otro, con lo que el hablante saldría beneficiado, en tanto en cuanto su imagen social se potenciaría ${ }^{9}$; o 2) intentar perjudicar (destruir incluso) la imagen del interlocutor, que interpreta la actividad como descortés, con lo que también se vería afectada, la del hablante mismo, aunque esto no siempre ${ }^{10}$.

Dependiendo de estos dos objetivos, se puede hablar de cortesía o de descortesía respectivamente, conceptos que no han de observarse como simples opuestos, sino como las dos caras de una misma moneda (la (des)cortesía). En este sentido, se ha llegado a ver la descortesía como:

fenómeno híbrido que comparte, en algunos contextos, rasgos en una línea de oposición a la cortesía, por ejemplo se puede explicar en relación a la cortesía cuando esta está ausente en situaciones en que sería esperable que se produjera; pero se presenta también en algunos contextos, como un sistema diferenciado de comportamiento social (Bernal 2007: 111).

9. Si se queda bien con el interlocutor, a la vez que se protege su imagen, el hablante queda en buen lugar. En eso consiste el equilibrio del que habia Hernández Flores (2004).

10. Su efecto es interpersonalmente negativo. Pero hemos de señalar que en las situaciones de conflicto, como el debate, por ejemplo, la descortesía, al ser la norma imperante, no desvaloriza generalmente la imagen del hablante, sino que, al contrario, este se erige en vencedor. 
Esto implica tener en cuenta factores como la interpretación que los propios interlocutores pueden hacer de los comportamientos verbales como (des)corteses en las distintas situaciones a las que se enfrentan, tal como han señalado Briz (1998), Eelen (2001) o Bravo (2002), quienes resaltan el papel del destinatario (objetivo de la (des)cortesía) en la evaluación del acto verbal en uno u otro sentido.

No se deben entender, por tanto, la cortesía y la descortesía como dos conceptos definitivamente opuestos, sino como aspectos que forman parte de una categoría única, la (des) cortesia, que puede mostrar sus distintas facetas en la interacción verbal, dependiendo de las necesidades comunicativas de cada situación. Este concepto, por ejemplo, explicaría comportamientos como el que observamos en uno de los teledebates que forma parte de nuestro corpus. Se trata del programa Lo que inTeresa ${ }^{11}$. Isabel San Sebastián, periodista conocida por su orientación política conservadora, le dice a un telespectador catalán, que interviene telefónicamente, que ella "ama al pueblo catalán", en un gesto de cortesía hacia aquel. Pero, inmediatamente, el telespectador, que defendía la autodeterminación de Cataluña y la aprobación del nuevo Estatuto para esta comunidad autónoma, le responde: "Pues muérase y deje de amarnos", porque ella, contrariamente a lo que pensaba este, interpretaba que la aprobación en el Congreso de dicho documento era una forma de romper España. Para ella, defender que Cataluña siguiera unida a España era una forma de mostrar amor hacia el pueblo catalán. Para el telespectador, era una manera más de mermar las libertades de ese mismo pueblo ${ }^{12}$.

Este tipo de reacciones son comprensibles porque, a menudo, desatendiendo los factores contextuales, se ha tenido una visión excesivamente positiva de la cortesía. Los recursos que identificamos de forma casi automática como corteses han sido relacionados, no en pocas ocasiones, con el fingimiento y la hipocresía, e incluso con conductas sociales calificadas de "falsas y represivas". De hecho, el propio Watts (2003) ha advertido que las estrategias de cortesía pueden ser, y

11. Programa de Antena 3, emitido en 2006 en horario de mañana, presentado por M. ${ }^{a}$ Teresa Campos, en el que bajo las formas de teletertulias y teledebates se trataban los más diversos temas de actualidad. En este caso nos referimos al emitido el 31/03/2006.

12. Asimismo, explicaría por qué, en un debate político, donde la descortesía es la pauta de comportamiento habitual (se trata, en términos generales, de afectar la imagen social del adversario), los halagos, catalogados habitualmente como estrategia de actividad cortés, llegan a desarrollar un efecto negativo de descortesía, pues, a menudo, se revisten de tintes irónicos, o así, al menos, lo valora el destinatario. 
han sido, asociadas al discurso hegemónico y de poder de las clases socialmente altas. Por lo tanto, no todo es acuerdo y armonía en lo que, desde lo codificado; denominamos cortesía ${ }^{13}$.

Esto ha hecho que muchos autores se replanteen la forma de concebir el fenómeno de la (des)cortesía. Y lo definen en términos de continuum. Este es el caso de Kienpointner (1997), para quien la dicotomía cortesía/descortesía debe ser sustituida por una visión de "continuum", en el que lo cortés o lo descortés sería una cuestión de grado, no de principios opuestos:

$\begin{array}{lll}\text { Total cooperation } & \text { Overpoliteness Cooperative } & \text { Total competition } \\ \text { Politeness } & \text { Rudeness } & \text { Rudeness }\end{array}$

The continuum of politeness and rudeness

(Kienpointner 1997: 258) ${ }^{14}$.

En este caso, la relación entre cortesía y descortesía se establecería en el eje "cooperación total $\leftrightarrow$ competitividad total". En medio de los dos polos extremos, se sitúan todos aquellos comportamientos que, dependiendo de factores sociales, culturales, situacionales, se identifican con la hipercortesía (resultado pragmático erróneo), la descortesía cooperativa (los casos de insultos rituales de Labov), la descortesía no cooperativa, motivada (la descortesía propiamente dicha, tipo insultos reales) o inmotivada (es el caso de los lapsus o las "meteduras de pata"). De acuerdo con esto, Kienpointner (1997) establece los siguientes rasgos de la (des)cortesía:

1) politeness or rudeness of utterances is a matter of degree; 2) rudeness is not simply derivable from politeness as a secondary phenomenom; 3) rudeness is a kind of prototipycally non-cooperative behavior, but it needs not always be (totally) irracional and or competitive behaviour; 4) likewise, politeness is a prototypically cooperative behaviour, but it needs not be always (totally) rational and there are types of less than opti-

13. "During the eighteenth century in Britain the term "polite', particularly when it was connected with language use, was manipulated in a socially selective way [...]. The concept of politeness was appropriated as the basis of a hegemonic discourse in which the ability to control a specific language variety was interpreted as providing access to high social status from which power could be exercised. Determining who was a member of 'polite society', however, was in the hands of those who had already gained access". (Watts 2003: 40)

14. Una visión similar a la mostrada por este autor es la que podemos encontrar en Mills (2003), Locher y Watts (2005) o Kaul de Marlangeon (2005). 
mally cooperative politeness; 5 ) only at a highly abstract level can concepts and strategies of existing politeness theories be considered to be universal (..); the relative importance and prominence of these concepts and strategies and their impact on the actual politeness or rudeness of utterances can only be judged relative to verbal and situational contexts, languages, and cultures (1997: 258-259).

Las referencias en este autor al tema central de esta contribución son claras: la variación como concepto esencial para el estudio de la (des)cortesía.

Es una constante que en los estudios sobre (des)cortesía se maneje toda una serie de conceptos en los que se refleja la necesidad de abordar esta función discursiva desde una perspectiva variacionista. Por ejemplo, el de cortesia codificada, cortesía interpretada y cortesía estratégica. La primera se vale de recursos lingüísticos más o menos convencionalizados (gracias, por favor, si me permite..., etc.). La segunda es evaluada en el devenir del discurso a partir de las reacciones de los participantes.

La cortesía estratégica se da al utilizar un recurso que habitualmente se identifica con un acto estratégicamente cortés para llevar a cabo un acto ulterior, que va más allá de la simple cortesía convencional. Es decir, usamos recursos corteses para conseguir algo más que "ser corteses". Por ejemplo, perdona es una forma que está codificada como cortés (se trata de un acto de reparación). Pero proferida con un tono más fuerte de lo normal, en una situación que se presupone tensa, estratégicamente está utilizada para señalar el desacuerdo con una situación, una actitud calificada como FTA (acto amenazante) para el otro. La cortesía estratégica es definida por Bravo (2005: 47) como:

un tipo de cortesía que tiene en muchos casos un bajo nivel de convencionalización y de codificación en la lengua, aunque posee un anclaje que hace que sea reconocida por los usuarios en su contexto. Este tipo de anclaje constituye un uso cuya interpretación es habitual en contextos similares. Se utiliza cuando se pretende realizar más de un objetivo (un acto a se hace pasar por un acto b).

Si analizamos el siguiente caso lo veremos más claro:

(1) HI- Nos habéis regalado el PERFUME DE ÚLTIMA HORA.

H2- LA BATIDORA QUE os dieron con la cuenta de ahorros.

H3- LA CENA ROMÁNTICA/ que os recomendó un amigo.

H4- Gracias // generosos. Pero ahora lo podéis arreglar.

H5- Porque ahora Línea Directa, si contratas un seguro, te REGALA LA MITAD DE OTRO. 
H6- Llamad porque eso SÍ NOS PARECE UN REGALO.

(spot de la marca Línea Directa, julio de 2007) ). $^{15}$

Aquí se utilizan dos recursos que, desde el ámbito de la cortesía codificada, se catalogarían como al servicio de una estrategia de cortesía: gracias es la fórmula prototípica que los hablantes utilizan para agradecer una acción o unas palabras. Ese generosos que sigue a la anterior fórmula responde a una estrategia de cortesía valorizante al estar resaltando rasgos considerados positivos en nuestra sociedad. Ambos recursos, utilizados irónicamente en este caso, dan lugar una actividad descortés basada en la estrategia que propone Culpeper (1996: 2005): "Sarcasm or mock politeness: the FTA is performed with the use of politeness strategies that are obviously insincere, and thus remain surface realisations", $(1996: 356)^{16}$.

Tanto la cortesia interpretada como la cortesía estratégica pueden ser extrapoladas también al ámbito de la descortesía. Se puede hablar de una descortesia interpretada cuando no hay intención clara de perjudicar con un acto de habla la imagen del otro, pero este sí lo interpreta así. De hecho, la descortesía, como veremos unos párrafos más adelante, depende en gran medida de la interpretación y valoración del interlocutor. Se puede ser estratégicamente descortés persiguiendo unos fines determinados. Por ejemplo, cuando tenemos alumnos en clase excesivamente pasivos, o que, por la hora de la clase, se encuentran apáticos, a menudo utilizamos estrategias altamente descorteses para que reaccionen. Aunque, en honor a la verdad, ese tipo de actividades necesita de un acto de reparación posterior, que lleve a una correcta interpretación de nuestro acto anterior, si no queremos caer en el peligro de ser malinterpretados. Algo así como ;hombre,

15. Las mayúsculas se utilizan para representar cadenas discursivas dichas en tono más alto o con mayor énfasis.

16. Este spot, además, es todo él un claro ejemplo de cómo a través de estrategias descorteses se intenta atraer al público. Se trata de un caso en el que, sirviéndose de los estereotipos que circulan en torno al hombre y su relación con su pareja, intenta ridiculizarlo, por medio del sarcasmo, para atraer al público femenino. Afecta a la imagen del hombre, como ser social, ridiculizando comportamientos estereotipados y fácilmente reconocibles. De esta forma, este anuncio se situaría en la frontera entre lo que Kienpointner (1997) llama sarcastic rudeness, una de las formas de la descortesía no-cooperativa, y la descortesía cooperativa: "it is posible to make a distinction between mildly ironic utterances, which have the goal of teasing the hearer in an amusing way and thus contributing to the mutual entertainement in a conversation, and bitingly sarcastic remarks, which hide a sharp attack against the face wants of the hearer under a seemingly polite surface". (264) 
si están vivos! En estos casos la actividad descortés funciona como un estímulo en busca de una reacción. De cualquier forma, todas las actividades (des)corteses producen en la interacción un efecto social, de carácter positivo, o efecto de cortesia $^{17}$, o negativo, de descortesía, el cual depende más de la situación en la que se dé el enunciado, que de cómo esté codificado el recurso lingüístico empleado ${ }^{18}$. Es lo que ocurre en el clásico "insulto de cariño". Y, de nuevo, nos topamos con la determinación de la situación en la interpretación de un recurso como (des)cortés.

De la mano de la sociología, en concreto de Goffman (1959 [2001] y 1967), llega a los estudios lingüísticos el concepto de imagen social (face), que se define como el valor social positivo que el individuo reclama para sí mismo durante la interacción con los otros. Y en esta "imagen" es también determinante lo que los otros interpretan acerca del valor social que ese individuo ha querido mostrar en dicho intercambio. Se trata, ante todo, de una proyección del yo ante el alter, un yo virtual, que se crea (que el hablante crea) a partir de la interacción social cotidiana. Por lo tanto, la imagen social tiene carácter público e interpersonal, pues depende de cómo el hablante, en nuestro caso, desee ser visto por los demás, a través de las relaciones comunicativas que establece. Para Goffman hay dos constantes en las interacciones: el auto-respeto y la consideración. Como parte integrante de un grupo, del hablante se espera que muestre respeto hacia él mismo y, a la vez, muestre consideración hacia las demás personas con las que se interrelaciona. El mantenimiento de la imagen propia y de la de los participantes en el intercambio comunicativo, condición sine qua non de la interacción, consiste en el mantenimiento de ambas constantes (auto-respeto y consideración).

En relación con este concepto, están los de imagen de afiliación e imagen de autonomía propuestos por Bravo (1999) ${ }^{19}$. En la conversación, según esta autora,

17. "Las atenuaciones constituyen actividades comunicativas tendentes a lograr disminuir el efecto social negativo de esos actos sobre la relación interpersonal y en la medida en que estas acciones hayan logrado sus objetivos hablamos de efectos de cortesia" (Bravo, 2002: 146).

18. En palabras de Bernal (2007), "hablaremos de efecto de cortesia y de efecto de descortesia como instancias no igualmente equiparables al efecto que habitualmente tengan determinados enunciados, de lo que se desprende que un enunciado que tenga un uso habitual descortés no tiene por qué ser recepcionado como tal, sino que puede interpretarse en un sentido diferente en la interacción en curso" (Bernal 2007: 22).

19. Estos conceptos ya fueron expuestos, aunque con otra terminología, por Fant (1989), quien habla de imagen inter-personal e imagen intra-personal, conceptos equivalentes a "afiliación" y "autonomía" respectivamente. 
la "autonomía" se manifiesta en todo lo que el individuo hace por diferenciarse del grupo, puesto que este desea mostrarse como alguien diferenciado del grupo (ideas propias, actitudes propias, etc.). La "afiliación", en cambio, se manifiesta en el comportamiento a través del cual el individuo intenta identificarse con el grupo. Para analizar los contenidos de imagen, Bravo $(1999,2001)$ propone partir de una serie de premisas culturales o convenciones sociales en función de los valores culturales vigentes en una sociedad que serían aplicables a un grupo concreto.

Por otro lado, está la imagen de rol, que desarrollan Fant y Granato (2002), Bravo (2004) o Fant (2007). El rol es un concepto de gran rentabilidad en el estudio de la (des)cortesía. Es, según Goffman (2001), una parte integrante de nuestra personalidad, de tal forma que el hecho de que un individuo represente el mismo papel en diferentes ocasiones ante una misma audiencia, hace que se desarrolle una relación social. De cualquier manera, el hablante no se puede entender como representante monoplano de un solo rol, sino que en sí mismo puede implicar uno o más papeles de contenidos variables (re)presentados por el actuante ante la misma o diferente audiencia de manera habitual. Por ejemplo, el rol de madre, el de profesora, el de amiga, etc. Todos ellos vienen definidos social, cultural y situacionalmente.

Veamos un ejemplo de salvaguarda de imagen de rol. Vamos a utilizar de nuevo la intervención de una popular conductora de programas tipo magazine en las distintas cadenas españolas. Se trata de M. ${ }^{a}$ Teresa Campos ${ }^{20}$. Esta ejerce de moderadora, pero en el nuevo estilo de moderación de los teledebates. Es decir, el moderador no se limita a repartir equitativamente los turnos de palabra, proponer tema, controlar el tiempo de exposición y reestablecer el orden si este se pierde, sino que además puede opinar sobre el tema, con el valor añadido de que, al ser moderador, el resto de los participantes va a respetar su turno, muchas veces usurpado a otros intervinientes, pues estos le reconocen su rol superior, y casi se dejan avasallar por ella.

Esta periodista en cuestión, además, se permite imponerse agresivamente y atacar tanto a intervinientes en el debate como a telespectadores que participan a

20. En esta ocasión hemos escogido un debate sobre el caso Malaya, que en esa fecha estaba en sus inicios (31/03/06) y sobre la aprobación en el Congreso de los Diputados del nuevo Estatuto para Cataluña. 
través del teléfono. Nadie se libra de sus mofas, de sus imposiciones, y, es más, de sus opiniones, que arroja como verdades absolutas que ha de respetar el resto.

Pero cuando ve que su imagen de rol, de moderadora, se está viendo amenazada, porque los demás participantes, al observar que su comportamiento es como el de uno más, pierden "el norte discursivo", reivindica su papel, a través de enunciados en los que hace valer su función. Incluso llega a ejercer una estrategia de descortesía a través del reproche, del que es ejemplo la siguiente intervención:

(2) Bueno yooo... sí que veo que como moderadora no me respetáis, porque este tema no e... Era para después. Estábamos con el tema de Marbella y [sic] Isabel, que viene con su teemaaa, esto de Marbella parece que no le interesa muuucho, y que quiere se... y que le interesa solo lo de (ininteligible). A nosotros nos interesa todo, toodo (Lo que inTERESA, A3, 31/03/06).

La moderadora apela a su papel, porque ha visto amenazada su imagen de rol. Y para defenderlo realiza una estrategia de contraataque asociando a los intervinientes a una actividad negativa (la del no respeto a los papeles sociales adjudicados) y luego dirige su comentario directamente a la participante a quien "otorga" toda la responsabilidad de ese acto de "no respeto", oponiéndola a todos los demás: "A nosotros nos interesa todo, toodo".

Junto a estos aspectos que pertenecen a lo que Bravo llama la imagen individual, se encuentra el concepto de imagen de grupo: la imagen del individuo como miembro o perteneciente a un grupo concreto: grupos profesionales, sociales (jóvenes, mujeres, etc.), familiares, etc. La imagen de grupo llega a ser tan importante que ya Goffman propugnaba que un miembro de un grupo debe evitar destruir la imagen de los otros miembros en prevención de la ruptura de relaciones dentro de dicho grupo. Asimismo, la conciencia de una imagen de grupo común hace que una actuación inadecuada de cualquiera de sus miembros ante personas pertenecientes a otro grupo resulte molesta a los otros miembros ${ }^{21}$.

21. Los hablantes llevan a cabo para dejar constancia de su pertenencia o no a grupos las llamadas actividades de imagen (face work). Son las acciones que la persona ejecuta para hacer corresponder su conducta con su imagen propia. Estas sirven para contrarrestar "incidentes" o eventos que pueden implicar una amenaza a la imagen y se conforman en prácticas habituales y estandarizadas, dentro de los grupos sociales. De esta forma, cada grupo social posee una serie de prácticas o habilidades sociales: técnicas de evasión, medidas defensivas, medidas protectoras, técnicas de corrección, de las cuales, obviamente, no nos podemos ocupar en este momento. Entre estas actividades de imagen se encuentran las actividades de (des)cortesía. 


\section{Variación y tipos de variación en el estudio de la (des)cortesía}

Hemos de hacer nuestras las palabras de M. Bernal (2007: 14) cuando dice:

no hay palabras o enunciados que sean inherentemente corteses o descorteses, ya que de ser así habrían de serlo en cualquier situación, y la prueba de ello es que incluso las fórmulas rutinarias de cortesía, fuertemente convencionalizadas, pueden tener una lectura de signo contrario ${ }^{22}$.

Este hecho hizo que nos planteáramos la tarea de defender aquí claramente un estudio variacionista para el análisis de las estrategias y recursos de (des)cortesía, pues de otro modo todo el esfuerzo que se está invirtiendo a nivel mundial en el estudio de esta categoría discursiva, se quedaría solo en un conjunto de estudios asistemáticos centrados individualmente en unos corpus concretos, a la par que aislados.

Esta propuesta no es nueva en los estudios sobre (des)cortesía. Han sido numerosas las voces que, en los últimos tiempos, se han alzado precisamente abogando por la necesidad de ampliar los aspectos implicados en la expresión lingüística del fenómeno de la (des)cortesía. Por ejemplo, Blum-Kulka (2002) y Mills (2003) nos expresan la necesidad de atender hechos como la percepción que el interlocutor tiene de la situación comunicativa, lo cual ayuda al análisis de las implicaciones de los enunciados en las actividades de (des)cortesía. Placencia y Bravo (2002) hacen hincapié en la necesidad de incluir, entre los factores que determinan la valoración de un acto de habla como cortés o no, la selección temática en la conversación.

Asimismo, son muchos los estudios donde, más o menos explícitamente, se pone de manifiesto esta necesidad de estudiar la (des)cortesía desde la variación. El simple hecho de que se estudie siempre en corpus cerrados, pertenecientes a grupos homogéneos de hablantes o de textos, nos habla de que existen normas de (des)cortesía tácitamente establecidas para cada uno de estos grupos y tipos discursivos. Por ejemplo, los estudios de las distintas modalidades de español de América en contraste con el español peninsular, no hacen sino hablarnos de la cortesía en relación con diferencias culturales y dialectales; los estudios que se

22. De ello han dado buena cuenta innumerables autores: Fraser (1990), Kienpointner (1997), Bravo (1996, 1999, 2002, 2003, 2004, 2005), Placencia y Bravo (2002), Spencer-Oatey $(2002,2003)$, etc. 
centran en grupos como amigos o familiares, o adolescentes masculinos, etc., nos hablan de la relación de este fenómeno con la variable social (generación, estrato social, etc. $)^{23}$; y los estudios dedicados a determinado tipo de textos y discursos, como el político, los mediáticos, los "tangos", la conversación, el español coloquial, etc., hacen clara referencia a la relación de la (des)cortesía y la variable diafásica. Nos estamos enfrentando a un fenómeno ligado íntimamente al contexto de usos de los recursos lingüísticos y a las condiciones socioculturales subyacentes en él.

La principal herramienta de análisis para poder llegar a aislar los comportamientos tanto corteses como descorteses será observar la repercusión que determinados usos lingüísticos tengan en la relación interpersonal a través del "efecto social" de cortesía-descortesía y lo relacionado con las actividades de imagen en general.

Veamos una serie de ejemplos que pueden ayudarnos a entender nuestra hipótesis de los condicionamientos de las variables diafásicas y diastráticas:

(3) H1.- Abuela, H2.- Hija, H3.- Nieta

$H 1$ se dirige a $H 3$, al enterarse a través de $H 2$ de que $H 3$ no ha asistido esa tarde a clase de Inglés, y le pregunta las causas. Hay que advertir que el tema es objeto de discusión habitual entre madre (H2) e hija (H3).

H1 (se dirige a H3).- Oye, me ha dicho mamá que hoy no has ido a Inglés. ¿Por qué?

H2.- Porque no le ha da(d)o la gana.

H3.- Por eso. Porque no me ha da(d)o la gana.

H2.- Oye, a la abuela no le contestes así.

H3.- Pero... si lo has dicho túuu.

Centrémonos en la expresión no me ha dado la gana. Según el Diccionario de uso de M. "Moliner, darle a alguien la gana de algo es "vulgar, rudo o usado con enfado" y significa querer. Asimismo, apunta a un uso negativo. ¿Por qué la madre de $\mathrm{H} 3$ recrimina a su hija el uso de esta expresión dirigida a la abuela $(\mathrm{H} 1)$, cuando, como responde $\mathrm{H} 3$, ya antes la había utilizado dirigida a la misma persona? El quid de la cuestión está en la consideración de la propia $\mathrm{H} 2$ hacia el acto

23. Zimmerman (1998) habla de la identidad o rol como factores de la interacción que pueden confluir en una situación y entre ellos refiere la edad, sexo, rasgos culturales, características de los interactuantes (amigos, compañeros, extraños, etc.). 
de habla que realiza ella y el que realiza $\mathrm{H}^{24}$. Y, cómo no, en el estatus social y, sobre todo, generacional de cada uno de los participantes.

La pregunta que realiza $\mathrm{H} 1$, tiene como alocutario y destinatario $\mathrm{H} 3$. Es a ella a quien las marcas lingüísticas señalan como tal. La respuesta que da $\mathrm{H} 2$, es, en realidad, una intromisión en una situación comunicativa, a la que en ese momento es, en cierta medida, ajena, y, por ello, más que la respuesta a una pregunta, es considerada como un comentario de alguien, que en ese momento es sólo auditorio, a la conducta de otra persona. En cambio, la respuesta de H3 sí es en sí misma la intervención reactiva de ese par adyacente, cuya intervención iniciativa es la pregunta misma. Es, por tanto, un acto de habla directo.

Esa construcción léxica (no darle a uno la gana de hacer algo) se da en registros informales, muy familiares, y en la modalidad coloquial. Viéndolo así, no debe haber ningún inconveniente en que se utilice en esta situación, dado que ya las relaciones entre abuelos, hijos y nietos en España hoy son más laxas que en otros tiempos. Pero debemos señalar que los contextos de uso de esta expresión no son generales, ni tan siquiera en situaciones como las señaladas aquí. Su uso depende del acto de habla que decidamos ejecutar. Y si bien es apta para describir comportamientos ajenos en conversaciones informales y familiares, no lo es tanto para describir los propios, y mucho menos para realizar un acto de respuesta a una pregunta. Es decir, desde el punto de vista de la función de (des)cortesía, el uso de esta expresión para responder es valorada como altamente descortés, incluso en situaciones de familiaridad.

La explicación puede estar en el propio significado de la expresión: según el $D R A E$, "en lenguaje poco culto, querer hacer una cosa" (s.v. gana). Es decir, es la expresión de la voluntad de alguien. Si describimos el comportamiento de otro, solo estamos describiendo. Pero si el sujeto de la voluntad es uno mismo, el hablante, en cierta manera impone un deseo de actuar en uno u otro sentido según las circunstancias. Por lo tanto, es, en cierta forma, una manera de imponerse, y en una respuesta a bocajarro, directa, como es el "porque no me ha da(d)o la gana" que analizamos aquí, es ruda e impositiva, porque se utiliza al mismo tiempo como mecanismo de cierre (se trata de zanjar la cuestión). De hecho, ante respuestas como estas la reacción del interlocutor es, en el mejor de los casos, el

24. Son las mismas palabras básicamente, pero no los mismos enunciados. Cambian los puntos de vista, los locutores, las intenciones, y los objetivos. 
silencio ${ }^{25}$. No olvidemos que una forma de demostrar que la imagen ha quedado afectada es "dejarle sin palabras".

Por ello $\mathrm{H} 2$, al valorar la respuesta como descortés, y, por tanto, como una amenaza a la imagen de $\mathrm{H} 1$ por parte de $\mathrm{H} 3$, realiza un acto reparador: la recriminación a $\mathrm{H} 3$ por una respuesta tan poco apropiada a las circunstancias. Pero, en relación a todo esto, hemos de advertir que la propia $\mathrm{H} 3$, antes de lanzar la respuesta, ha llevado a cabo un acto atenuativo, señalando la respuesta de $\mathrm{H} 2$ como válida: por eso. Porque no me ha da(d)o la gana. De algún modo, evita adjudicarse toda la responsabilidad de la respuesta, haciéndose partícipe de lo dicho anteriormente. Pero no ha calibrado suficientemente que su acto de habla no es el mismo.

Nos merece especial atención también la recriminación de $\mathrm{H} 2$ hacia $\mathrm{H} 3$ por la respuesta. Nos podemos preguntar ¿por qué ese acto reparador de una tercera persona en la situación comunicativa? Y es aquí donde entra el factor generacional en juego.

Antes dijimos que al igual que en el terreno de la cortesía se buscaba el equilibrio social de los participantes en el acto comunicativo, en la descortesía también se produce un cierto equilibrio, aunque esta vez basado en la afectación de la imagen. Es decir, cuando alguien, por medio de actividades de descortesía, afecta la imagen social del otro, ese alguien no queda impune, sino que su imagen queda igualmente afectada, en sentido positivo o negativo. Con la respuesta de $\mathrm{H} 3$, la imagen de $\mathrm{H} 1$ ha quedado afectada, y eso se manifiesta en el silencio de esta, que no puede reaccionar ante la contundencia de la intervención. Al mismo tiempo, la imagen de $\mathrm{H} 3$ también se ve socialmente afectada: se presenta como una persona ruda, excesivamente directa, e impositiva, poco apropiada lingüisticamente. De ello se da cuenta $\mathrm{H} 2$, madre de $\mathrm{H} 3$, que ve cómo en esa respuesta se ve comprometida su imagen de rol, su imagen de buena madre que, en este caso, no ha sabido educar a su hija para que se comporte de forma adecuada lingüísticamente. Es esto lo que realmente mueve a $\mathrm{H} 2$ a realizar esta reprobación. $\mathrm{H} 2$ apela al estatus de abuela de HI, un grado que va a regular el uso verbal: a pesar de los mayores niveles de tolerancia lingüística que existen hoy entre padres, hijos y abuelos en relación con tiempos pasados, aún existen las llamadas barreras generacionales.

25. Probablemente, la respuesta verbal a este acto daría lugar a una confrontación e, incluso, a una ruptura de relaciones. 
De ahí que los usos lingüísticos que hoy son permitidos, por razones de cercanía, solidaridad, afiliación, entre padres e hijos en contextos no conflictivos ${ }^{26}$, aún no son permitidos entre estratos entre los que la distancia social, que no afectiva, es mayor. Por lo tanto, es la madre, en su rol, quien ha de ser la que repare, porque es o ha sido su responsabilidad enseñar contextos y situaciones de uso adecuados para los distintos recursos lingüísticos. Lo que para algunos estratos es un recurso que se encamina a la afiliación, en otros se torna en un recurso de carácter absolutamente descortés.

Otro caso que nos puede servir de ejemplo de cómo las variables de tenor inter personal influyen en el la percepción de los recursos lingüísticos como corteseso descorteses, es el siguiente:

(4) $\mathrm{H1}$ (mujer) y $\mathrm{H} 2$ (hombre) son compañeros de trabajo con igual rango jerárquico. El intercambio se produce ante otro grupo de compañeras, de estrato jerárquico laboral más bajo, con los que HI tiene un trato más habitual y cercano que H2. H1 se acaba de enterar por otros compañeros de la inminencia de una situación laboral nueva para H2, que ella desea para si misma. Lo cual le provoca eso que llamamos "envidia sana".

H1.- ¡Joder! Conque te han concedido lo del sabático. Y yo sin enterarme de que lo habías pedido. ¡Qué guarro! ¡Qué mamón! Y yo sin saberlo. ¿iY te vas a ir por ahí y me dejas aquí con to(d)a esta gente!? ¿Qué mamón!

H2.- (Sin dejar de sonreír, aunque de forma un tanto forzada) Pos sí.

H1.- ¡Qué mierdorro, tío!

H2.- (En tono más bajo, y al oído de H1) Oye, córtate un poco. ¿No te importa que esta gente pueda pensaaar...? No sé. Es que no me siento cómodo diciéndome estas cosas delante de ellas. ¿Tú sí?

H1.- Son de confianza, hombre. Pero bueno... Perdona. Ya no lo hago más.

H2 teme, al no tener ese valor de [+cercanía] con el grupo auditorio, que su imagen social y de rol (estatus laboral más alto) queden afectadas ante la posibilidad de que este esté valorando esa "sarta" de palabras que, normativamente, están codificadas como insultos, actos amenazantes por excelencia. H1 está utilizando este recurso como una estrategia de cortesía al estar realizando una actividad de

26. Otro valor muy distinto tendría esa respuesta en una situación de conflicto extremo, como una discusión. Entre padres e hijos adolescentes suelen usarse, como recursos de afiliación en contextos no polémicos ni conflictivos, palabras malsonantes y apelativos calificados como insultantes desde la lógica de la norma adulta. Estas mismas serían ofensivas y amenazantes en contextos de conflicto con las mismas personas. 
imagen de afiliación al grupo, intentando neutralizar las fronteras jerárquicas en esa situación comunicativa. Ambos, $\mathrm{H} 1$ y H2, están manejando variables distintas a la hora de interpretar, de valorar, como cortés o descortés, la actividad realizada por H1. Por otro lado, el hecho de que el auditorio pertenezca a un estrato jerárquico laboral inferior, también determina la percepción negativa de $\mathrm{H} 2$. Por lo tanto, ya no se trata solo de la percepción que tiene el "insultado" acerca de la intencionalidad del hablante, sino de la percepción que tiene aquel sobre la percepción que de esos actos tengan otros, y que pueden poner en peligro su imagen de rol, e, incluso, su imagen de jerarquía, según terminología de Fant (2007).

En este ejemplo, se ha producido un "fracaso pragmático" [pragmatic failure, en terminología de Thomas (1983)], pero no por desconocimiento de las normas y códigos sociales, sino por la ausencia de un contexto completamente compartido entre los interlocutores. Se están manejando contextos divergentes (el de los compañeros de escala más baja en $\mathrm{H} 2$, y el de amigos y colegas en $\mathrm{H} 1$ ), y eso hace que los recursos para mostrar afiliación que utiliza H1 parezcan inadecuados a H2. Para H2 se está produciendo una "descortesía inmotivada" [inmotivated rudeness de Kasper (1990) y Kienpointner (1997)]. Por lo tanto, como vemos, las claves contextuales de esta interacción (relación vivencial de proximidad o no) son fundamentales para catalogar estos actos como descorteses o no. La lectura que el destinatario hace en este caso de lo que realmente son insultos lúdicos o retóricos determina en qué términos va a seguir desarrollándose la interacción. De hecho, aquí derivó hacia unos usos lingüísticos menos marcados que, a juicio de nuestro H2, eran más adecuados a la situación, y le hacían sentir más cómodo.

Otro ejemplo es el siguiente anuncio de la campaña de verano de 2007 del producto PepsiMax:

(5) Los compañeros de Comunicación del Juampi le dedicamos esto.

Mira, Juampi, tu corto titulado El Silencio nos parece una ñapa. Además, estamos un poco hartos de que vayas de visionario del cine. Que estamos en primero, tío. ¿No te das cuenta de que todo el mundo se descojona de ti? Déjate ya de ir por la calle así, mirándolo todo como si fueras un director. Y vuelve con nosotros al fútbol siete. ¿Tas entera(d)o, (mijo)?

Como vosotros, quizás no nos creamos los más listos. Quizás no nos haga falta. Pepsi Max. Máximo sabor sin azúcar.

La estructura "estar un poco + adjetivo", utilizada en una situación de normalidad, es decir, donde la cortesía es la norma, la búsqueda del equilibrio entre los 
interlocutores es el objetivo primordial de las intervenciones de ambos dispositi vos comunicativos, para atenuar nuestras opiniones o contribuciones con el objetivo de no imponernos. Por ejemplo:

(6) A- ¿Vienes al cine?

B- Estoy un poco cansada / Estoy cansada.

Las respuestas de $\mathrm{B}$ son, en ambos casos, un rechazo a una invitación; es, por tanto, una respuesta no deseable para el acto ilocutivo de invitación que se ha lleva do a cabo. Pero, mientras la primera respuesta de B deja abierta la posibilidad de una reformulación de la respuesta, en el sentido de que permite la reparación de la posible amenaza a la imagen de $\mathrm{A}$ al ser rechazada su propuesta, la segunda, al ser tajante, elimina dicha posibilidad de reparación en caso de conflicto extremo En la primera se produce la atenuación semántico-pragmática del elemento "har to", mediante la modificación morfológica externa de "un poco".

Frente a esto, el ejemplo del spot presenta una situación diametralmente distinta. Se trata de una situación de conflicto. Lo que se está realizando es una reprensión en toda regla, un acto de reproche y sanción a las actitudes del pró tagonista de este anuncio, "el Juampi". En esta situación, que tiene como fin último dañar, si no destruir, la imagen del protagonista, es extraño que se em pleen recursos que se encaminen a reparar la imagen del otro, como son los atenuativos. Es más, es esa extrañeza que produce en el receptor, y en los restantes destinatarios (casi auditorios de este discurso), ese choque entre el valor codificado de ese elemento y la situación de conflicto abierta, lo que hace que se in vierta el valor contextual de este elemento, y se potencie una fuerza ilocutiva mayor del resto de la secuencia, a favor de la sanción agresiva, y no de la reparación de las imposiciones.

Véase, si no, el contraste que ofrece esta secuencia frente a otra posible sin elementos atenuativos:

(7) Además, estamos hartos de que vayas de visionario del cine.

El "estar hartos" se diluye entre los restantes recursos de imposición, a pesar de su aparente mayor agresividad en este sentido. Es el contraste, el extrañamiento de la utililización de una fórmula ligada generalmente a una situación de equilibrio, lo que le dota de ese valor intensificador en estas situaciones polémicas. Además, el hecho de que en un tipo de habla caracterizado por lo directo de sus 
manifestaciones, sobre todo en su registro más coloquial, como es este caso, hace que se perciba la atenuante como una fórmula de intensificación.

Otro ejemplo es el que presenta el siguiente spot publicitario:

(8) H1- Nosotras no intentamos aparcar en el hueco de una bici.

H2- Nosotras no recorremos media calle marcha atrás.

H3- ¡CONDUCIMOS MEJOR QUE ELLOS! Y lo vamos a probar.

H4- Si eres mujer, tienes que llamar.

H5- Porque ahora Línea Directa asegura por 199 euros a los buenos conductores.

H6- ¡NOSOTRAS!

H7- Cuantas más llamemos, MÁS CLARITO les va a quedar.

(Línea Directa, julio de 2007)

Dos son los valores que se le han adjudicado tradicionalmente al diminutivo: por un lado, su valor cuantitativo en referencia a la disminución de tamaño; por otro lado, su valor afectivo-emotivo, que implica connotaciones de afecto, cariño, $\operatorname{etc}^{27}$.

Desde una perspectiva pragmática, se reconocen los siguientes valores del diminutivo:

-cuantificación: minoración-empequeñecimiento objetivo.

-modalidad-argumentación: destacar un objeto, realce informativo, afectivo o atenuativo. En este último sentido, podríamos tener una atenuación cortés o una atenuación argumentativa, con valores de inversor argumentativo, apelativo, eufemístico, sobrerrealizante o irónico ${ }^{28}$.

En Fuentes-Alcaide (2004: 1080) se señala que el diminutivo, en algunas ocasiones, "aumenta la fuerza argumentativa del sustantivo. Sería un sobrerrealizante, tal como señalan J. Portolés (1998) y M.M. Negroni (1995)", de tal forma que, en estas situaciones este potencia la dimensión argumentativa del elemento afectado (modificado) por este morfema. En nuestro ejemplo, con el diminutivo, que apa-

27. Incluso A. Alonso (1967: 164-5) reconoció en el diminutivo un valor cortés, no impositivo, en los diminutivos utilizados en enunciados de claro valor imperativo: vaya despacito. De todas formas, la mayoría de los autores que se han ocupado de este elemento establece el valor de familiaridad como el principal.

28. Cfr. Fuentes-Alcaide (2004: 1077). 
renta atenuar, se ha conseguido realizar una intensificación. Y esto se ha produci do precisamente por encontramos en una situación de conflicto abierto, en la que no es en absoluto esperable una estrategia atenuativa. De nuevo, el tenor interpersonal condiciona el valor estratégico de un recurso, diseñado, al parecer, para llevar a cabo una acción contraria a la que realmente se realiza ${ }^{29}$.

Otro elemento que coadyuva aquí para invertir el valor atenuativo en intensificador es el recurso fónico de intensidad o tono, que como afirma A. Briz (1998), puede "convertir otros formalmente atenuados, corteses, en empleos intensificados, en apariencia descorteses" (144). Esto es lo que puede Ilegar a explicar los valores que estos elementos atenuativos han tenido en los ejemplos analizados.

\section{Hacia una nueva visión de la (des)cortesía}

Todo lo dicho nos ha llevado a replantearnos una serie de aspectos en relación con la (des)cortesía. Por ejemplo, ¿en qué consiste la hipercortesía (supercortesia, overpoliteness, etc.)?

La hipercortesía, tal y como se ha tratado hasta ahora, se definía como un exceso de cortesía. De hecho, Kerbrat-Orecchioni (2004) la define como: "presencia de un marcador excesivo en relación a las expectativas normativas vigentes" (49). Luego, en el vector desarrollado por Kienpointner (1997) se situaría, a nuestro modo de ver, a la izquierda incluso del concepto de cortesía, no en un lugar intermedio entre la cortesía y la descortesía, puesto que el hablante que se muestra hipercortés busca una cooperación excesiva, tomando uno de los ejes vertebradores del continuum de Kienpointner, que en realidad puede volverse en su contra. Es un "ir más allá" de la cortesía, es manifestarse "más que cortés", "cortés en exceso". Por lo tanto, esa imagen de la cortesía y la descortesía como los dos polos de un continuo se nos desfigura, porque habría un paso más hacia la izquierda de la misma en la que estaría enclavada la hipercortesía.

Pero aún hay más datos que nos hacen replantearnos esta visión. En muchas ocasiones, sobre todo, si la hipercortesia es "estratégica" y, además, se da en una situación de [+ distanciamiento, - familiaridad] (pensamos ahora en el típico

29. El valor intensificador del diminutivo también ha sido señalado por M. Albelda (2007: 54), aunque no señala su valor descortěs. 
dependiente de una tienda o de un concesionario de coches que se muestra en extremo cortés, e incluso servil; que en su afán de ayuda, se incluye como persona del discurso en una conversación ajena, etc.), esta llega a rozar los efectos de los mecanismos de descortesía, pues nos encontramos en una situación en la que no nos está permitido enfrentarnos frontalmente con el hablante hipercortés, sin caer nosotros mismos en la descortesía (es decir, no nos podemos defender fácilmente de este fenómeno $)^{30}$. Nos preguntamos cómo un fenómeno que está en el extremo opuesto puede llegar a tener contacto con el otro extremo. Esto es fácilmente entendible si consideramos este fenómeno como un continuo verdaderamente "no interrumpido", en el que no podemos distinguir los polos extremos porque están intimamente relacionados, hasta tal punto que, como en la filosofia oriental, los opuestos pueden llegar a tocarse.

Por todo ello, debemos plantearnos una nueva consideración de la (des)cortesía:

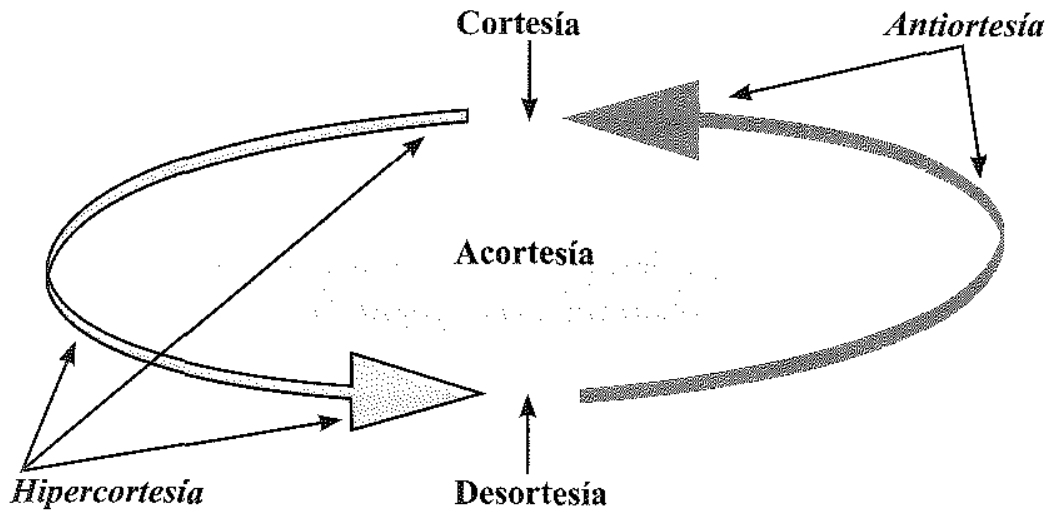

Desde este punto de vista, cualquier acto de habla, cualquier enunciado, puede ser situado en un punto de este proceso a mayor o menor distancia de la cortesía o descortesía, teniendo en cuenta la codificación, la intencionalidad del hablante

30. El problema de la "hipercortesía" es que siempre se ha visto como un fallo pragmático debido a la falta de adaptación de hablantes de una lengua a otra, o hablantes de una misma lengua pero pertenecientes a distintas culturas (cfr. Kienpointner, 1997: 257). No se ha contemplado desde el punto de vista estratégico: "hacer la pelota", por ejemplo. De cualquier forma, aun en el caso de que se tome como un comportamiento involuntario, se nos hace difícil situarlo en un lugar en el que se baja el grado de cooperación discursiva y se tiende a la competitividad. 
y la interpretación del oyente. En ese posicionamiento, intervendrán como factores determinantes variables como la distancia/cercanía social de los interlocutores, variables culturales, generacionales, etc. De tal forma que un mismo recurso lingüístico puede ser utilizado/interpretado como cortés o descortés dependiendo de todos estos factores.

Pero podemos decir más. La hipercortesía y la anticortesía no son, a nuestro juí cio, fenómenos al mismo nivel que la cortesía o la descortesía. Lo que se ha dado en llamar hipercortesia y anticortesía no es más que el uso de recursos lingüís. ticos que, desde el punto de vista de la cortesía codificada, se están utilizando estratégicamente para realizar actividades de cortesía o de descortesía. Es decir, son formas de utilizar recursos que se han catalogado desde el punto de vista del código.

Realmente, desde nuestra perspectiva, la (des)cortesía se nos antoja como un pla. no cuyos puntos cardinales contrapuestos son la cortesía y la descortesía. Dependiendo de la situación en la que nos encontremos, nuestra aguja apuntará a usos que, bien por nuestra intencionalidad, bien por la interpretación, o bien por ambas cosas a la vez, apuntarán hacia un punto u otro, acercándonos a uno u otro. Por ejemplo, habrá situaciones en las que por la relación de proximidad entre los interactuantes, por el rol que desempeñan, etc., lo que denominamos hipercor tesía se acerque a lo que entendemos como cortesía, búsqueda de la armonía y equilibrio. Pero en otras ocasiones, en las que los factores situacionales no son los mismos, esto se convertirá en una actividad que estará más cerca de la descortesía, porque realmente estamos "molestando", "afectando" la imagen del otro, que realmente puede no llegar a sentirse cómodo en la interactuación (el dependiente extremadamente solícito).

\section{Conclusiones}

Una vez más podemos demostrar que, en la valoración de determinados recursos como corteses o descorteses, son la variable social y la diafásica (estilística) las que en realidad se imponen, dando lugar a interpretaciones corteses o descorteses de los recursos lingüísticos utilizados. Por lo tanto, se reafirma la necesidad de una perspectiva variacionista en el estudio de la (des)cortesía verbal, no solo basada en lo etnográfico o cultural, sino, dentro de la misma cultura, en las variables sociales, geográficas y contextuales-situacionales, en interrelación, al igual que en cualquier otro aspecto del uso de las lenguas. 
La situación comunicativa concreta en un determinado contexto sociocultural es la que va a definir como corteses o descorteses, y también anticorteses, los recursos lingüísticos utilizados como estrategias de (des)cortesía.

En este sentido, la utilización de materiales de apoyo como son los tests de hábitos sociales, se ha manifestado de gran rentabilidad para la investigación del uso del lenguaje en relación con, por ejemplo, el contexto sociocultural de los usuarios.

Lo que en estos momentos se nos impone es la sistematización de los resultados de todos esos estudios que se están haciendo teniendo en cuenta implícitamente todas las variables sociales, culturales, contextuales y dialectales, para no quedarnos en un cúmulo de estudios y análisis atomistas o encerrados en las cárceles de los corpus.

\section{Referencias bibliográficas}

ALBELDA, Marta (2007): La intensificación como categoría pragmática: revisión y propuesta. Frankfurt a. M.: Peter Lang.

Alonso, Amado (1967): "Noción, emoción, acción y fantasía en los diminutivos", en: Estudios lingüisticos. Temas españoles. Madrid: Gredos, 161-189.

BERNAL, María (2007): Categorización sociopragmática de la cortesia y de la descortesía. Un estudio de la conversación coloquial española. Stockholm: Stockholm University.

BiUM-KULKA, Soshana (1990): “'You don't touch lettuce with your fingers': parental politeness in family discourse", en: Journal of Pragmatics 14, 259-289.

- (2002): "Pragmática del discurso", en: Van Dijk, Teun A. (ed.): El discurso como interacción social. Barcelona: Gedisa, 67-99.

Bravo, Diana (1999): “¿Imagen positiva vs. imagen negativa? Pragmática sociocultural y componentes de face", en: Oralia 2, 155-184.

- (2001): "Sobre la cortesía linguística, estratégica y conversacional en español", en: Oralia 4, 299-314.

- (2002): “Actos asertivos y cortesia: Imagen de rol en el discurso de académicos argentinos", en: Placencia, M. ${ }^{a}$ Elena/Bravo, Diana (eds.): Actos de habla y cortesía en español. München: Lincom Europa, 141-174.

- (2003): "Actividades de cortesía, imagen social y contextos socioculturales: una introducción", en: Bravo, Diana (ed.): Actas del I Coloquio del Programa EDICE. Stockholm, 98-108 (www.edice.org).

- (2004): "Tensión entre universalidad y relatividad en las teorías de la cortesía", en: Bravo, Diana/Briz, Antonio (eds.): Pragmática sociocultural: estudios sobre el discurso de cortesía en español. Barcelona: Ariel, 15-37.

- (2005): "Categorías, tipologías y aplicaciones. Hacia una redefinición de la "cortesía comunicativa", en: Bravo, Diana (ed.): Estudios de la (des)cortesía en español. Ca- 
tegorias conceptuales y aplicaciones a corpora orales y escritos. Stockholm-Buenos Aires: Editorial Dunken, 21-52.

Briz, Antonio (1998): El español coloquial en la conversación. Esbozo de pragmagramática. Barcelona: Ariel.

Brown, Penélope/Levinson, Stephen (1987): Politeness. Some Universals in Language. Cambridge: Cambridge University Press.

Cordrsco, Ariel (2003): “Afiliación y desafiliación: contexto sociocultural en el análisis de la interrupción y de sus consecuencias en la interacción", en: Bravo, Diana (ed.): Actas del I Coloquio del Programa EDICE. Stockholm, 150-163 (www.edice.org).

CULPEPER, Jonathan (1996): "Towards an anatomy of impoliteness", en: Journal of Pragmatics, 25, 349-367.

- (2005): "Impoliteness and entertainmen in the television quiz show: The Weakest Link", en: Journal of Politeness Research 1,35-72.

EELEN, Gino (2001): A critique of politeness theories. Manchester: St. Jerome.

FANT, Lars (1989): "Cultural mismatch in conversation: Spanish and Scandinavian communicative behaviour in negotiations settings", en: Hermes Journal of Linguistics 3, 247-264.

— (2007): "Rapport and identity management: A model and its application to Spanish dialogue", en: Placencia, M. ${ }^{a}$ Elena / García, Carmen (eds.): Research on Politeness in the Spanish-Speaking World. London: Lawrence Earlbaum Associates, 335-365.

- Granato, Luisa (2002): "Cortesía y gestión interrelacional: hacia un nuevo marco conceptual", en: SUS Working Papers $I V$ 1, Stockholm University (www.ispla.su.se/ iis/\#SIIS).

Fraser, Bruce (1990): "Perspective on Politeness", en: Journal of Pragmatics 14, 219-236.

- NoLEN, Walter (1981): "The association of deference with linguistic form", en: Inter national Journal of Sociology of Language 27, 93-109.

Fuentes Rodríguez, Catalina/AlCAide LARA, Esperanza (2004): "La dimensión argumentativa del diminutivo", en; Villayandre Llamazares, M. (ed.): Actas del V Congreso de Lingüistica General. León 2002. Madrid: Arco-Libros. 1073-1085.

GOFFMANN, Irving (1967): Interaction Ritual: Essays on Face-to-face Behaviour. New York: Doubleday Anchor Books.

- (2001): La presentación de la persona en la vida cotidiana. Buenos Aires: Amorrortu.

GRice, H. Paul. ([1967] 1975): "Logic and conversation", en: Cole, Peter/Morgan, Jerry L. (eds.): Syntax and Semantics 3: Speech Acts. New York: Academic Press, 41-58.

HERNANDEZ Flores, Nieves (2002): La cortesia en la conversación española de familares y amigos. La buisqueda del equilibrio entre la imagen del hablante y la imagen del destinatario (Tesis doctoral). Aalborg: Aalborg Universitet/ Institut for Sprog og Internationale Kulturstudier, 37.

- (2004): "La cortesía como búsqueda del equilibrio de la imagen social", en: Bravo, Diana/Briz, Antonio (eds.): Pragmática sociocultural: estudios sobre el discurso de cortesía en español. Barcelona: Ariel, 95-107.

KASPER, Gabriele (1990): "Linguistic politeness. Current research on issues", en: Journal of Pragmatics, 14, 193-219.

Kaul de Marlangeon, Silvia (2005): "Descortesía de fustigación por afiliación exarcebada o refractariedad. El discurso tanguero de la década del '20'", en: Bravo, Diana 
(ed.): Estudios de la (des)cortesia en español. Categorias conceptuales y aplicaciones a corpora orales y escritos. Stockholm-Buenos Aires: Editorial Dunken, 299-318. KeRBrat-Orecchion, Catherine (1996): La conversation. Paris: Seuil.

- (2004): “¿Es universal la cortesía?”, en: Bravo, Diana/Briz, Antonio (eds.): Pragmática sociocultural: estudios sobre el discurso de cortesía en español. Barcelona: Ariel, 39-53.

KIENPOINTNER, Manfred (1997): "Varieties of rudeness. Types and functions of impolite utterances", en: Functions of Language 4-2, 251-287.

LAKOFF, Robin (1973): "The logic of politeness: or minding your p's an q's", en: Papers from the $9^{\text {th }}$ Regional Meeting of the Chicago Linguistic Society. Chicago: Chicago University Press, 92-305.

LEECH, George (1983): Principles of Pragmatics. London: Longman.

LOCHER, Miriam A./WATTs, Richard (2005): "Politeness theory and relational works", en: Joumal of Politeness Research 1, 9-33.

Meier, Ardith J. (1995): "Passages of Politeness", en: Journal of Pragmatics 24, 381-392. MiLls, Sandra (2003): Gender and Politeness. Cambridge: Cambridge University Press.

ROMANE, Suzanne (1996): El lenguaje en la sociedad. Una introducción a la sociolingüistica. Barcelona: Ariel.

Placencia, M." Elena/Bravo, Diana (2002): "Panorámica sobre el estudio de los actos de habla y la cortesía lingüística", en: Placencia, M. ${ }^{a}$ Elena/Bravo, Diana (eds.): Actos de habla y cortesia en español. München: Lincom Europa, 1-19.

SPENCER-OATEY, Helen (2002): "Rapport management: A frame work of analysis", en: Spencer-Oatey, Helen (ed.): Culturally speaking: managing rapport through talk across cultures. London: Continuum, 11-45.

- (2003): "Developing a framework for non-ethnocentric 'Politeness' research", en: Bravo, Diana (ed.); Actas del I Coloquio del Programa EDICE. Stockholm, 86-96 (www. edice.org).

THomas, Jenny (1983): "Themes of interaction", en: Sinclair, J.M./Brazil, D. (eds.): Teacher Talk. Oxford: Oxford University Press, 91-112.

WatTs, Richard (2003): Politeness. Cambridge: Cambridge University Press.

ZIMMERMAN, Don (1998): "Identity, context and interaction", en: Antaki, C./Widdicombe, S. (eds.): Identities in talk. London: Thousand Oaks, 87-106. 